\title{
Animated Construction of Chinese Brush Paintings
}

\author{
Fan Tang, Weiming Dong \\ Xiaopeng Zhang,
}

\author{
Yiping Meng, Xing Mei, Feiyue Huang, \\ Oliver Deussen
}

\begin{abstract}
In this paper, we present a method for reconstructing the drawing process of Chinese brush paintings. We demonstrate the possibility of computing an artistically reasonable drawing order from a static brush painting that is consistent with the rules of art. We map the key principles of drawing composition to our computational framework, which first organizes the strokes in three stages and then optimizes stroke ordering with natural evolution strategies. Our system produces reasonable animated constructions of Chinese brush paintings with minimal or no user intervention. We test our algorithm on a range of input paintings with varying degrees of complexity and structure and then evaluate the results via a user study. We discuss the applications of the proposed system to painting instruction, painting animation, and image stylization, especially in the context of art teaching.
\end{abstract}

Index Terms-Chinese brush painting, animation, drawing analysis, art teaching.

\section{INTRODUCTION}

C HINESE brush painting is a unique and fascinating form of art that has been around for more than 1500 years. Owing to the difficulty in understanding the proper creation of paintings, instructional videos have been created to show the sequential application of strokes during the drawing process. Commercial movies and advertisements often use animation sequences of dynamic paintings for special effects. Video scribing, the animated production of large images for educational purposes, is a new art form that explicitly uses drawing animation. Such dynamic painting videos are typically created from a camera recording of the application of strokes during the manual creation of a painting. The recording functionalities of drawing software are also utilized. However, these methods are laborious, and they limit the creation of dynamic paintings to a few professional painters.

These problems motivated us to develop an image-based method for creating painting animations. Large numbers of brush paintings have been created over the years, and they serve as great sources for constructing animated drawings. Given a static painting, we intend to estimate a drawing order for the strokes that is approximately consistent with painting principles and is artistically reasonable for educational or commercial applications (see Fig. 1).

The basic principles of Chinese brush painting (cf. [1], [2]) typically demand a solution for the following key problems (e.g., Fig. 2): (i) given an input painting with clearly defined

- F. Tang and Y. Meng are with NLPR, Institute of Automation, Chinese Academy of Sciences, Beijing, China and University of Chinese Academy of Sciences, Beijing, China. E-mail: \{tangfan2013, mengyiping2013\}@ia.ac.cn.

- W. Dong, X. Mei, and X. Zhang are with NLPR, Institute of Automation, Chinese Academy of Sciences, Beijing, China. E-mail: $\{$ Weiming.Dong, Xing.Mei, Xiaopeng.Zhang\}@ia.ac.cn.

- F. Huang is with YouTu Lab, Tencent, Shanghai, China. E-mail: \{garyhuang\}@tencent.com.

- O. Deussen is with University of Konstanz, Germany. E-mail: \{oliver.deussen\}@uni-konstanz.de strokes, a model should be built to represent strokes with a set of features; (ii) an overall structure should be built for stroke positioning. The drawing process can be roughly divided into three stages: outlining structure, drawing local details, and adding decorative strokes. This process adheres to the principle of "division and planning", i.e., to organize compositions by placing and arranging their elements [3]; (iii) the drawing direction for each individual stroke should be determined, and the animation should be rendered by following the stroke order.

Performing art varies with people and differs over time. Although artists generally follow certain principles because of some conventions [4], the drawing process is individual and involves personal preferences. This fact implies that multiple solutions might be available for our problem. Therefore, our objective is to find a reasonable solution that is consistent with the laws of art construction instead of searching for the best drawing order of strokes.

We propose an effective mechanism for estimating such a reasonable order, provided that we have a set of $2 \mathrm{D}$ strokes extracted from a Chinese brush painting that consists of clearly defined strokes. Our work makes the following contributions:

- Introducing the problem of reconstructing and animating the drawing process of Chinese brush paintings.

- Summarizing artistic guidelines for ordering strokes from art principles.

- Effectively simulating the drawing process by constructing a multi-stage structure and optimizing the stroke orders by natural evolution strategies (NES) [5]; encoding both the visual similarity of strokes, such as color and contour, and the variation of strokes during painting, including ink wetness, ink thickness, and shape.

As the estimated drawing processes might be subjective due to the variability of artistic principles, we conduct a user study to evaluate the perceived quality of our solutions 

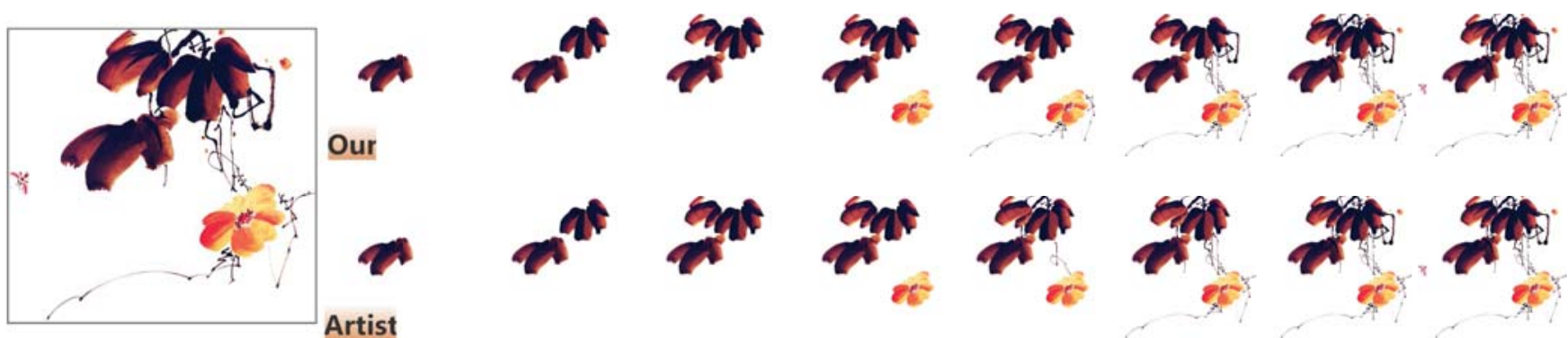

Fig. 1. Guided by painting principles, we derive an artistically reasonable order of an input static Chinese brush painting (left) to automatically animate the drawing process (top right). A user study shows that the reconstructed order is comparable to the stroke order interactively specified by an artist (bottom right). The animations can be used as instructional videos in fine art education or to create special visual effects in commercials.

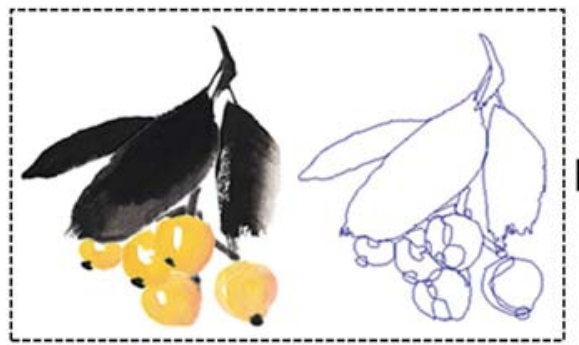

(a) Input: a painting with cleanly defined strokes

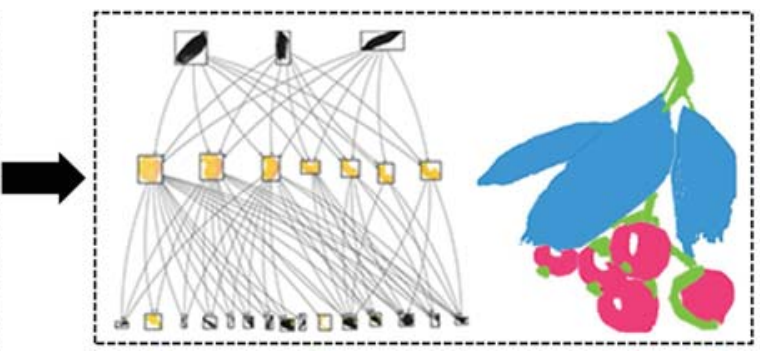

(b) Roughly organized strokes into multi-stages

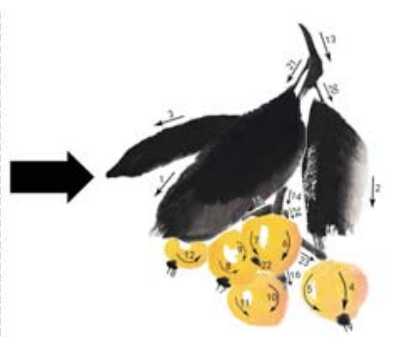

(c) Optimize stroke orders

Fig. 2. Global structure of the painting process. (a) Input a painting with cleanly defined strokes. (b) The strokes of different stages are organized into different layers of the Hasse diagram (left) and are depicted by different colors (right). (c) The optimized drawing order of the strokes as indicated by their numbers and stroke directions by the corresponding arrows.

compared with that of alternative strategies. A quantitative evaluation is also performed by comparing our results with manually generated drawing processes from artists. We analyze and evaluate a variety of Chinese brush paintings and conceptually apply our technique to drawing education, storytelling, and commercial visual effects.

\section{Related Work}

Related works come from a number of fields in computer science, cognition and arts. We focus on the technical works in these fields.

Art canons. Instructional books of Chinese brush paintings often introduce the basic principles of drawing order [1], [2], [6], especially for specific objects [7]. Chinese painters do not use sketches or models but paint from memory according to their own impressions of objects. In contrast to other techniques, brush painting cannot be improved, corrected, or fixed [8]. The most important part of a painting is always done first, and the rest is done afterwards in the order of importance. From the first to the last stroke, the artist must "get it right". Therefore, drawing order becomes one of the key elements in creating a good Chinese brush painting. Furthermore, common drawing theories consider the order of strokes in a painting as a reflection of the mental organization of a scene and its hierarchical relationships [9]. Note that although digital paint systems (e.g., MoXi [10]) can achieve various realistic effects of ink dispersion, such systems focus on using physical simulation to enhance the final drawing rather than reconstructing the intermediate states of a drawing process.
Recovering drawing process. Perceivers of manually created artifacts are able to extract order information from static images [11]. Hays and Essa [12] decompose a photograph into a plausible arrangement of brush strokes by using a library of brush stroke textures. Flagg and Rehg [13] design an interactive system around a set of interaction modes that assist artists in painting ordered sets of layers that comprise finished paintings. Monroy et al. [14] reconstruct and visualize the temporal order of the drawing process of medieval images. Fu et al. [15] reveal the sketching sequence of a line drawing by mapping the gestalt and cognition rules to computational procedures. Liu et al. [16] additionally integrate common drawing phases and achieve plausible results with an entropy-based ordering strategy. Mao et al. [17] present a region-based method to extract the structure lines within cartoon images; this method is helpful in recovering drawing process of cartoons. Given the richer features of a brush painting stroke compared to a line, our problem is more complex than the recovery of the drawing process of line drawings. Yang et al. [18] order all the brush strokes in a painting sequence according to the average gray value of each brush stroke. We demonstrate that this simple strategy often fails to generate satisfactory results for many images. Tan [19] present a set of techniques to decompose a time lapse video of a painting into a sequence of translucent stroke images. However, the current work focuses on reconstructing the drawing process from a static image rather than a dynamic video.

Analyzing and animating Chinese artworks. Techniques for analyzing and constructing animations from input paintings or calligraphy can improve the reusability of artistic 


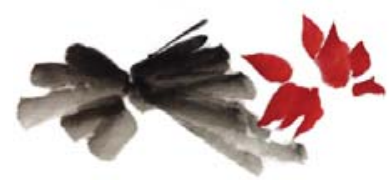

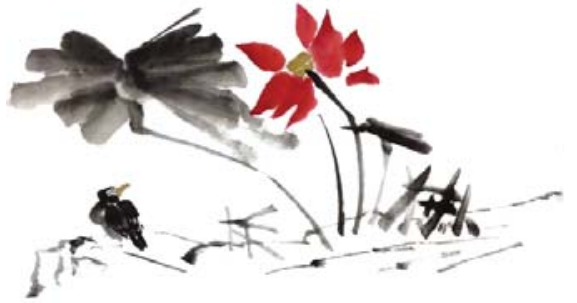

(b) Addition of strokes of local details.

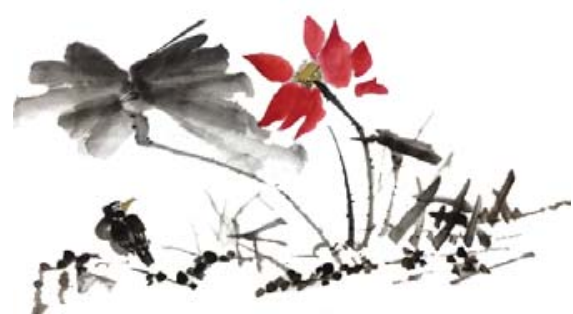

(c) Addition of decorative strokes.

Fig. 3. Creating a painting in multiple stages. Painters usually draw elements in a primary-local-decorative manner.

works. Xu et al. [20] decompose a given input painting into hypothetical strokes and animate the painting at the brush stroke level. Their method for automatically recovering separate, vectorized brush strokes is useful in solving our problem and can be used as a pre-processing step in our system. Zhang et al. [21] synthesize animations of running water in the style of Chinese paintings for applications such as cartoon making. Yang et al. [22] estimate brush trajectories with shape outlines and dynamically render them into brush strokes by mapping footprint textures sampled from input images. This technique is useful for the analysis and appreciation of Chinese ink paintings. Yang et al. [23] decompose a calligraphic character into several strokes and construct writing rhythm and brush footprint models to dynamically render calligraphy in the oriental ink style.

Non-photorealistic rendering (NPR). Synthesizing brush paintings from images and 3D models has been extensively studied [24]. Amati and Brostow [25] digitize and transform Chinese ink paintings into 2.5D sprites. Wang and Wang [26] suggest a mathematical model with a physical base to synthesize an image with color ink diffusion. Xie et al. [27] model a brush as a reinforcement learning agent and apply this brush agent to automatically convert photographs to oriental ink-style paintings. Xu et al. [28] present a strokebased approach for creating real-time ink-wash paintings of geometric models. Dong et al. [29] abstract images with the help of saliency maps and then convert them into Chinese ink paintings through non-physical ink diffusion processes.

Painting robots. Machines for simulating the human painting process have been manufactured since the 19th century. Proponents of modern computer and robot-based approaches include Yao and Shao [30], who let a Chinese painting robot draw bamboos. The drawing orders and control points of strokes are specified manually. Lindemeier et al. [31] evaluate painterly rendering techniques that work within a visual feedback loop of eDavid, a painting robot [32]. The strokes are dynamically generated and drawn one by one without following artistic principles. Sun and $\mathrm{Xu}$ [33] develop a calligraphy robot capable of writing a large piece of aesthetic calligraphic work. Our work could easily be coupled to these machines to create authentic Chinese paintings.

\section{OVerview}

Input and stroke modeling. Two main techniques exist for creating Chinese paintings: meticulous (Gong-bi) and freehand (Xie-yi). Meticulous paintings incorporate delicate calligraphic strokes and a close attention to detail, whereas the freehand style emphasizes the interpretive aspect of the brushwork. Freehand style also seeks to express the essence of the subject rather than the details of its appearance. In freehand paintings, a single stroke can produce astonishing variations in tonality, from deep black to silvery gray. Our system only considers paintings that employ brush strokes economically, that is, paintings with cleanly defined strokes (small freehand style), and excludes brush paintings created by splash ink or drawn in meticulous style. in which individual strokes are difficult to distinguish. Our rationale behind this decision is that beginners learning Chinese painting always start from small freehand paintings to train their basic painting skills. Thus, animating the construction process of small freehand paintings is more useful for teaching than animating other paintings. In addition to Chinese paintings, our system can work with Sumi-e and certain watercolor paintings. We consider an input painting to be an image composed of an unordered set of already segmented 2D brush strokes. We then build a model that describes stroke properties by using a set of features (Section 5.1) that incorporates geometric and shading characteristics.

Multi-stage structure. In general, Chinese brush paintings are created in the following way (Fig. 3). First, primary strokes determining the composition of the painting are painted. These strokes often occupy large areas and are drawn by dark ink. Then, the painter progressively refines the painting by adding strokes for local details. Finally, some dry or thin strokes are drawn, and splattered or dotted ink is applied to decorate the painting.

This observation motivates us to first construct a representation that consists of a primary-local-decorative order(Section 5.2). This representation serves as a stageby-stage drawing order, paves the way for introducing of multi-stage drawing guidelines from artistic principles, and provides a meaningful structure of input strokes.

Stroke ordering. A drawing order of all the strokes is estimated in the second step. Following the guiding principles given in Section 4.2, we formulate an energy function that encodes both the multi-stage structure of the painting and the rules in stroke ordering (Section 5.3). We then find the optimal solution for the energy function to compute the stroke order by using NES (Section 5.4).

Animation. Given a stroke order of the input painting, the last step is to reproduce the painting process using the optimized stroke order. The direction for each individual stroke is determined according to the guidelines of the stroke direction (Section 4.3). A flood-filling-based method is used 
to render the strokes (Section 5.5).

\section{GUIDELINES FOR STROKE ORDERING}

Chinese brush paintings emphasize simplicity, flowing brush strokes and graceful design, using black ink and the three primary colors. We obtain the original guidelines by carefully studying and analyzing paintings in instructional books and videos and by consulting with professional artists and lay painters with drawing training experience. In this section, we identify important guidelines related to the geometry, shading, and color features of strokes.

\subsection{Stage structure of a drawing}

As mentioned above, in freehand Chinese paintings, artists attempt to capture the essential nature or character of a subject and not its actual physical appearance; every area of the painting has to be interesting to the eye [8]. In general, painters tend to draw elements in a primarylocal-decorative manner, which follows the order of the contribution of elements to the content of the painting. They first add important strokes that determine the composition of a painting and then move on to strokes that depict local details, finally using splattered or dotted ink to decorate the scene.

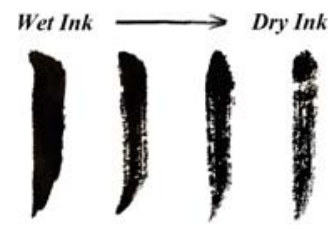

(a) Strokes of different wetness

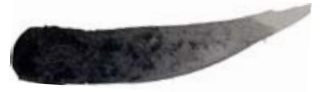

(c) Thickness variation within a stroke

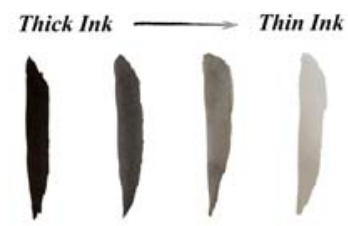

(b) Strokes of different thicknesses

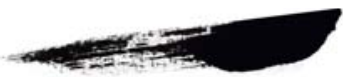

(d) Wetness variation within a stroke
Fig. 4. Stroke styles and the variations of main stroke properties such as wetness and thickness.

\subsection{Revealing stroke order}

The stylized expression of shade and texture is the most important brush technique in Chinese painting. The strength and quality of ink and water create various numerous variations. Thick ink is deep and glossy when brushed onto paper or silk, whereas thin ink gives a lively, translucent effect. Light and darkness, texture, weight, and coloring can be conveyed simply through the thickness of the ink. In addition, ink can be used dry or wet. The charm of a brush-and-ink painting comes not only from the artist's intentional self-expression but also from the interaction of the ink and water with the textured paper. Fig. 4 shows the different stroke styles used in Chinese brush paintings. In our framework, such visual features of strokes are crucial to reconstructing the stroke order.

Although the division into stages can roughly describe the overall creation of a brush painting, the stroke order should still be determined. Stroke order is affected by a wide spectrum of factors including geometry, shading,

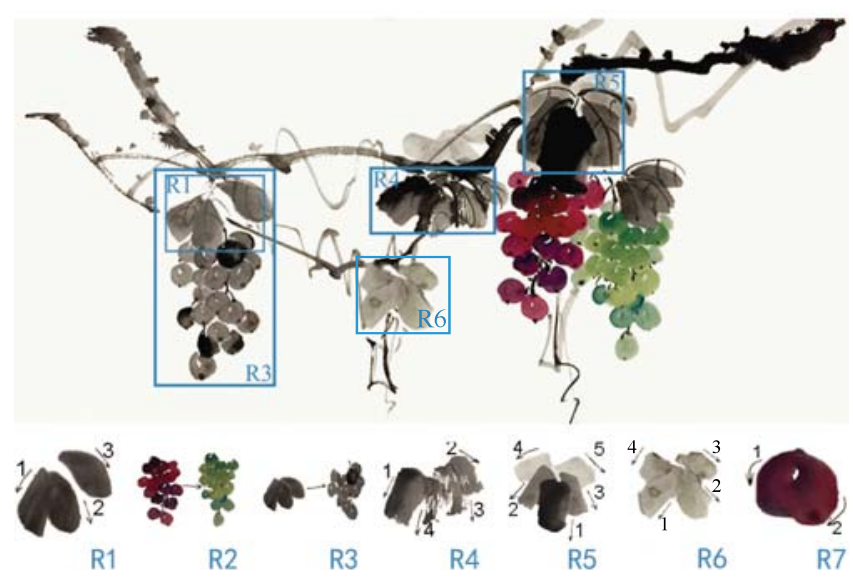

Fig. 5. Guidelines for ordering strokes: (R1) by proximity; (R2) by color similarity, in which fruits in the same color are drawn together; (R3) by contour similarity, in which the geometrical shapes of the leaves and fruits are different and the strokes of similar contours are added together; (R4) by wetness variation; (R5) by thickness variation; (R6) by shape variation; and (R7) shows a rounding object represented by two strokes. The order is shown with the numbers.

color, semantic, and motoric reflexes [1], [2], [7]. Although semantic features and drawing convenience can impose important ordering constraints, especially for some specific objects (e.g., insects), we mainly focus on the visual aspects. Guidelines for stroke ordering (see Fig. 5) can be classified in three scales: (i) inter-stroke-based rules indicating that strokes in close Proximity or with similar Color/Shape are likely to be added together; (ii) individual stroke-based rules indicating the local priority of strokes with respect to Wetness, Thickness and Size; (iii) a composition-based rule describing a specific technique when the objects being painted are Rounding. The details are as follows.

R1 (Proximity): Proximity is an important issue in determining order because painters often like to move to an adjacent element to reduce time and effort (see Fig. 5 (R1)).

R2 (Color): To create a Chinese brush painting, an artist needs to use several different brushes to draw strokes of different styles and sizes and in different colors. Thus, to facilitate the drawing process, the artist usually groups strokes of similar colors in the drawing process by drawing one group with one brush and then to change the brush to draw another group. Therefore, we consider strokes with similar colors to be drawn together (see Fig. 5 (R2)).

R3 (Shape): Similar to the grouping according to color similarity, the artist usually groups strokes of similar geometrical shapes in the drawing process. Thus, strokes with similar contour shapes are usually drawn together (see Fig. 5 (R3)).

R4 (Wetness): A fact about Chinese brush painting is that each brush stroke is a defining move that draws up a portion of the painting, which is neither improved upon nor corrected. No sketch is prepared, and no model is used. The artist paints with rapid, mentally constructed strokes transporting a "mind image" to mulberry paper. Thus, during the drawing process, the artist usually draws multiple strokes with the same inked brush, for which these sequential strokes may change from wet to dry, i.e., in a local region, wet strokes are likely to be added prior to dry strokes 
(see Fig. 5 (R4)).

R5 (Thickness): Similar to wetness, strokes with thick ink in a local region are likely to be added prior to strokes with thin ink (see Fig. 5 (R5)).

R6 (Size): To progressively add strokes in multiple stages, artists prefer to draw strokes with visually important sizes first according to their area, length, and scale in the painting process (see Fig. 5 (R6)). Strokes of different shapes are usually created by different types of brushes (brushes with different heads).

Note that when keeping two specific rules under observation, conflict arises among individual rules in the guidelines. Such conflict is quite likely to occur during the drawing process. The reason is that guidelines R1-R6 belong to a type of "soft" constraints that act as a whole. They "vote" for the final result. Aside from such soft constraints, we also define the rule of rounding, which is a hard constraint.

R7 (Rounding): In the Chinese painting process, a round object (e.g., grape, eye) is often represented by two strokes. The start point of one stroke is extremely close to the start point of the other stroke and vice versa for the two end points. Therefore, these two strokes are almost invariably drawn together (see Fig. 5 (R7)).

\subsection{Stroke direction and starting location}

After a stroke order is determined, the painting direction for each stroke is identified. Unlike line drawings for which directional preferences depend on the convenience of the mechanical movement toward the subject's wrist and fingers as well as the visibility of existing strokes, no universal principle exists for the direction of strokes in Chinese brush paintings. As a matter of fact, stroke directions may be distinctively different for different objects. Nevertheless, the direction of each stroke from a static painting image could still be estimated. Typically, the variation in moisture and ink content caused by the motion of the brush reveals the stroke direction to a certain extent. Our guidelines on stroke direction are summarized as follows.

D1 (Wetness preference): Whenever a distinct difference in ink wetness exists between the two skeleton points of a stroke (see Fig. 4(c)), we draw the stroke from the skeleton point with wet ink to the one with dry ink.

D2 (Thickness preference): Whenever a distinct difference in ink thickness exists between the two skeleton points of a stroke (see Fig. 4(d)), we draw the stroke from the endpoint with thick ink to the one with thin ink. The calculation of the stroke skeleton points is described in Section 5.1 .

The last open problem is from which stroke we have to start the painting:

S1 (Starting location): For Chinese brush paintings of small freehand style, the starting location is usually related to the object to be painted. In our system, the optimization process automatically decides the starting stroke.

\section{Methodology}

In this section, we map the guidelines summarized in the previous section to our computational procedures. Our system accepts a set of unordered brush strokes extracted from a painting as input. We develop an interactive tool for decomposing a given painting into single strokes by using approaches given in [20] and [22]. For complex inputs with overlapping or indistinct strokes, we manually refine the segmentation results. This process typically takes less than three minutes of user interaction. Fig. 6 shows an example of stroke decomposition of a simple bird painting.
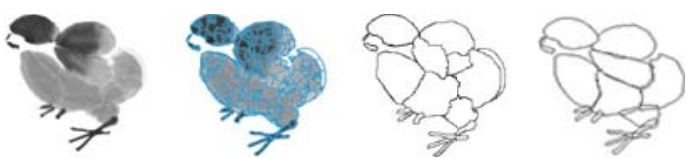

Fig. 6. Stroke decomposition of a simple bird painting.

\subsection{Stroke model}

Building a feasible model to describe stroke properties is crucial for stroke ordering. We propose a set of features involving shape, color and texture to model a brush stroke and denote $\mathcal{S}=\left\{s_{i} \mid i=1,2, \ldots, N\right\}$ as the set of strokes.

F1 (Skeleton points): We adopt the skeleton point extraction model of Tang et al. [34]. For each stroke, we first use the Canny detector to detect edges and compute the morphological gradient image (Fig. 7(a)). Then, we use the Harris corner detector to obtain the corner points and use the pairs of points with the largest geodesic distance [35] as the skeletal points of a stroke $\left(\vec{X}\left(s_{i}\right)\right)$. Thus, we define the spatial distance $D\left(s_{i}, s_{j}\right)$ between two strokes as the minimum distance between the skeletal points of both strokes. Aside from $D\left(s_{i}, s_{j}\right)$, we define $D^{\prime}\left(s_{i}, s_{j}\right)$ as the smaller the distance between the other two skeleton points; a small value equates to a high possibility for the two strokes to form a round object in the painting.

F2 (Length): We define the geodesic distance between skeletal points as the length $L\left(s_{i}\right)$ (see Fig. 7(a)).

F3 (Area): We define the area of a stroke $A\left(s_{i}\right)$ as the number of valid pixels in $s_{i}$. We treat a pixel as a valid pixel if it is not white (the $\mathrm{Y}$ value of the pixel color using the YIQ model is less than 240).

F4 (Scale): We adapt the method in [36] to calculate the scale of a stroke, denoted as $S\left(s_{i}\right)$. In the definition from the literature, stroke $s_{i}$ encompassing region $R$ denotes the existence of at least one location to put $R$ completely inside $s_{i}$, denoted as $R \subseteq s_{i}$. With this relation, we define the scale of stroke $s_{i}$ as

$$
S\left(s_{i}\right)=\arg \max _{t}\left\{R_{t \times t} \mid R_{t \times t} \subseteq s_{i}\right\},
$$

where $R_{t \times t}$ is a $t \times t$ square region. i.e., our stroke scale is defined as the largest square that a stroke region can contain (see Fig. 7(b)).

F5 (Shape): We adapt Discrete Fourier Transform (DFT) to extract the contour information as the shape of a stroke. When a 1D DFT is applied to the vector defined by the coordinates of the boundary points of a stroke (Fig. 7(c)), the sequence $\widetilde{U}$ of $\mathrm{M}$ complex numbers is transformed into an M-periodic sequence of complex numbers, expressed as $\widetilde{F D}_{\mu}\left(s_{i}\right)$ with $\left(-\frac{M-1}{2}<\mu<\frac{M-1}{2}\right) . \widetilde{F D}_{\mu}$ is the original Fourier Descriptor (FD) of a stroke. However, measuring the similarity of two contours by FD is computationally 


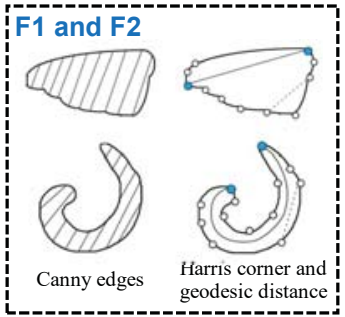

(a) Skeleton points and length

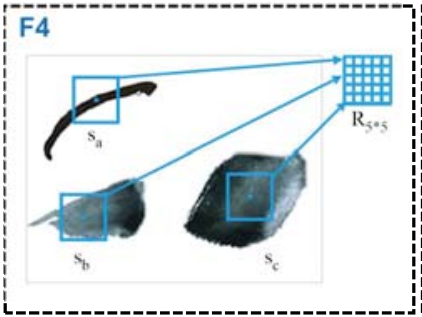

(b) Scale: the largest square that a region can contain

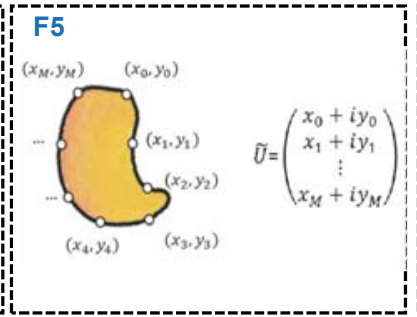

(c) Coordinates of the points along the circumference, clockwise ordered.

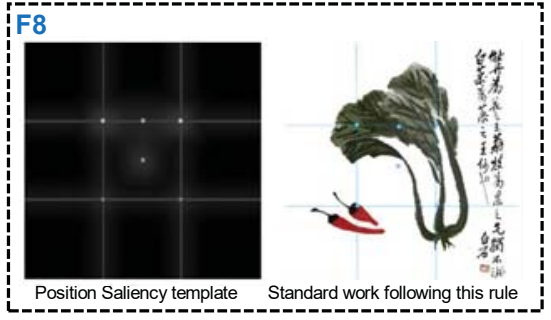

(d) Position saliency: lookup-table feature extraction

Fig. 7. Some features used to construct the stroke model.

expensive. Therefore, the original coefficients are combined to roughly describe the contour information of a stroke. We define the circularity $F_{c i r}$ of a stroke as

$$
F_{c i r}\left(s_{i}\right)=\frac{\widetilde{F D}_{1}\left(s_{i}\right)}{\sum_{\mu=1}^{\frac{M-1}{2}}\left(\left|\widetilde{F D}_{-\mu}\left(s_{i}\right)\right|+\left|\widetilde{F D}_{\mu}\left(s_{i}\right)\right|\right)},
$$

and define the slenderness $F_{\text {sle }}$ of a stroke as

$$
F_{\text {sle }}\left(s_{i}\right)=1-\frac{\left|\widetilde{F D}_{1}\left(s_{i}\right)\right|-\left|\widetilde{F D}_{-1}\left(s_{i}\right)\right|}{\left|\widetilde{F D}_{1}\left(s_{i}\right)\right|+\left|\widetilde{F D}_{-1}\left(s_{i}\right)\right|} .
$$

A large $F_{\text {cir }} \in[0,1]$ means that the stroke is orbicular, whereas a large $F_{\text {sle }} \in[0,1]$ means that the stroke is tenuous. Circularity and slenderness jointly depict the shape of the stroke.

F6 (Ink wetness and thickness): As mentioned in Section 4.2 and shown in Fig. 4, the combination of ink and wash results in an attractive Chinese brush painting. Ink wetness measures the amount of the water on the brush. Low wetness of a stroke means the stroke is hollower than the strokes in wet ink. We define the feature of ink wetness as the percentage of valid pixels (defined in F3) in the polygonal approximation of the stroke's external profile, denoted as $W\left(s_{i}\right)$. Ink thickness is measured by the average Y value $G\left(s_{i}\right)$ of all the valid stroke pixels and is defined as $T\left(s_{i}\right)=255-G\left(s_{i}\right)$. Note that the wetness of a brush, stroking force, and drawing velocity are not solved in the equation. Hence these two features are only mathematical estimations of ink wetness and thickness.

F7 (Color): We define stroke color as the average color of the stroke pixels (using the RGB model), denoted as $C\left(s_{i}\right)$.

F8 (Position saliency): In Chinese painting, foremost strokes are usually placed in salient positions. This principle is called "start and end at the three-seven point", which is similar to the rule-of-thirds principle in photography. We use the template put forward by [37] to estimate position saliency. This template defines a 2D (300) lookup table that returns a score $P\left(s_{i}\right)$ given the location of the stroke centroid. Light areas have a high score, and blurred locations that are close but not exact have good scores (see Fig. 7(c)).

In our experiments, all features are normalized to $[0,1]$.

\subsection{Multi-stage structure}

One of the important principles of Chinese painting is "division and planning", which involves a progressive organization and placement of strokes. Thus, we first analyze the input set of strokes and organize them into a hierarchical structure, with the stroke properties being the fine-scale features that are grouped together by functional characteristics to capture the global structure of the input painting (see Fig. 2). From top to bottom, the hierarchy serves as a multi-stage drawing order of the input painting. A natural drawing order starts from the outlining of the principal part by adding large strokes with thick ink in salient locations, progressively introducing strokes that depict local details, and finally adding decorative strokes that are often thin and dry. Thus, we define a partially ordered relation over the stroke set $\mathcal{S}$ so as to roughly divide strokes into different categories. Such relation essentially determines the global priorities of strokes according to their features.

The drawing categories of two strokes are considered different if their features are distinctly different. We define a partial ordering relation $\preccurlyeq$ for two strokes $s_{i}, s_{j} \in \mathcal{S}(\mathcal{S}$ being the set of strokes):

$$
\text { iff } \operatorname{sum}\left(\overrightarrow{s_{i}}-\overrightarrow{s_{j}}\right)>\epsilon \text {, then } s_{j} \preccurlyeq s_{i},
$$

where $\vec{s}$ is a vector composed of the stroke features F2-F4, F6, and F8 (features are equally weighted), $\epsilon=2.0$ in all our experiments. The relation $\preccurlyeq$ is a strict irreflexive partial order and $\langle\mathcal{S}, \preccurlyeq\rangle$ is a partially ordered set, which can be illustrated by a directed acyclic graph $\mathbb{G}(\mathcal{S})$. The edge of the graph denotes the existence of a drawing priority between the two strokes.

We further simplify $\mathbb{G}(\mathcal{S})$ into a Hasse diagram $\mathbb{H}(\mathcal{S})$ [38]. The nodes in $\mathbb{H}(\mathcal{S})$ represent the elements in $\mathcal{S}$ and are sorted from bottom to top by following their orders in $\mathbb{G}(\mathcal{S})$. Specifically, for any two strokes $s_{i}, s_{j} \in \mathcal{S}$, if $s_{j} \preccurlyeq s_{i}$, then $s_{i}$ is arranged on the top of $s_{j}$. If $\left\langle s_{i}, s_{j}\right\rangle \in \preccurlyeq$, and $\forall s_{k} \in \mathcal{S}, \nexists s_{k} \preccurlyeq s_{i} \wedge s_{j} \preccurlyeq s_{k}$, we add an edge from $s_{i}$ to $s_{k}$. Fig. 8 shows the visualization of a Hasse diagram $\mathbb{H}(\mathcal{S})$. A reasonable stage structure is constructed by considering the aforementioned mentioned stroke features. The strokes on the top level are usually added in the first stage, whereas the ones on the bottom represent the second and third stages. In other words, the strokes on the top are likely to be drawn before the bottom ones. However, the diagram can only roughly group strokes into different stages; they cannot represent the full stroke order. The algorithm for refining the local orders of the strokes is defined in the following sections.

\subsection{Formulation of stroke ordering}

An optimized drawing order should express the multi-stage painting process and satisfy the local order constraints. Thus, rather than separately ordering strokes at each level as done in [15], we formulate the ordering problem as a global 


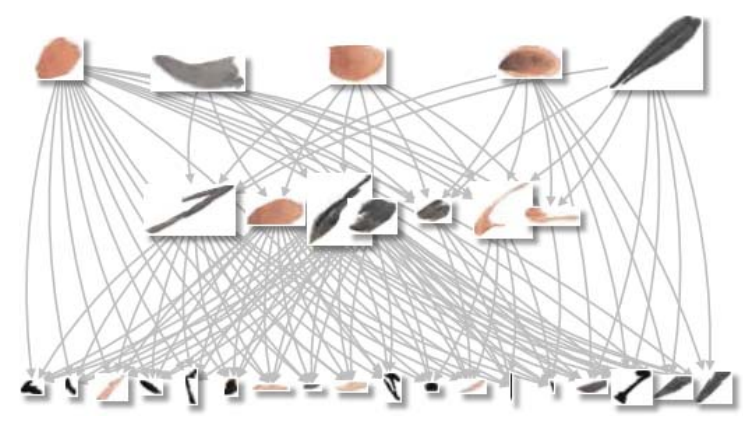

Fig. 8. Hasse diagram simplified from the directed acyclic graph. The diagram represents the global structure of an input painting related to the rough drawing stages.

energy minimization problem over a permutation ordering of index set $\mathcal{I}=[1,2, \ldots, N]$ ( $N$ is the number of strokes) with a corresponding energy that is minimized over all the permutations $\mathcal{P}$ of $\mathcal{I}$. We denote $p$ as one permutation, $p_{i}$ as the index of the $i$ th stroke in $p$, and $s_{p_{i}}$ as the $i$-th stroke in $p$.

Topological sorting. We establish the initial stage-level drawing order of strokes by performing a topological sorting [39] on the Hasse diagram $\mathbb{H}(\mathcal{S})$. In our implementation, the node with the maximum outgoing degree is always selected because a large outgoing degree equates to a large number of nodes on the bottom that support the corresponding drawing priority. Note that [34] also orders strokes on the basis of a topological structure of a graph, but the formulation of the graph only considers "prime" strokes.

Energy function. Denote the drawing order obtained from the topological sorting as $p^{*}$ and the target optimized order as $p^{\prime}$. We formulate the ordering problem as the following global optimization problem:

$$
p^{\prime}=\arg \min _{p \in \mathcal{P}} c(p)+\left(1-d\left(p, p^{*}\right)\right),
$$

which is subject to additional constraints derived from detected round objects (which will be described later). Here $c(\cdot)$ is the stroke cost item that encodes the guidelines in Section 4.2, and $d(\cdot)$ is a difference function used to ensure that the final order is also consistent with the multi-stage structure (described later). The sequence of $\left(s_{p_{1}^{\prime}}, s_{p_{2}^{\prime}}, \ldots, s_{p_{N}^{\prime}}\right)$ gives us the desired ordering of $\mathcal{S}$.

We formulate the stroke cost in Equation (2) as

$$
\begin{array}{r}
c(p)=\sum_{i=1}^{N} \sum_{j=1, j \neq i}^{N} \eta(i, j) \cdot\left[\omega \cdot c_{\text {cons }}\left(s_{p_{i}}, s_{p_{j}}\right)\right. \\
\left.+(1-\omega) \cdot c_{\text {var }}\left(s_{p_{i}}, s_{p_{j}}\right)\right],
\end{array}
$$

where $c_{\text {cons }}$ captures the order-insensitive properties of an individual stroke $s$ (e.g., color, contour and spatial location), $c_{v a r}$ evaluates the order-sensitive variation cost between stroke $s_{i}$ and $s_{j}$, and $\omega$ is a weighting term for balancing the influence between the two terms. The function $\eta(i, j)$ is a monotonically decreasing window function that encourages the sorting of lines in local regions. We let $c(p)$ accumulate the cost between two strokes only when their rank difference in the current permutation is less than $R$ :

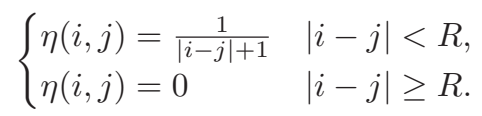

Here, a large $R$ makes the energy function powerful because it can affect a large number of strokes. However, it can also increase the computational complexity. We set $R=4$ in all our experiments.

Consistency cost. The consistency cost $c_{\text {cons }}$ measures the visual similarity of a sequence of strokes, according to their features R1-R3. We evaluate $c_{\text {cons }}\left(s_{p_{i}}, s_{p_{j}}\right)$ of strokes $s_{p_{i}}$ and $s_{p_{j}}$ according to their contours (F5), colors (F7) and their distance (F1) using the Euclidean distance:

$$
c_{\text {cons }}\left(s_{p_{i}}, s_{p_{j}}\right)=\left\|\vec{s}_{\text {cons }}\left(p_{i}\right)-\vec{s}_{\text {cons }}\left(p_{j}\right)\right\|+D\left(s_{p_{i}}, s_{p_{j}}\right),
$$

where $\vec{s}_{\text {cons }}\left(p_{i}\right)=\left(F_{\text {cir }}\left(s_{p_{i}}\right), F_{\text {sle }}\left(s_{p_{i}}\right), G\left(s_{p_{i}}\right)\right)$ is a vector that is composed of stroke features $\mathbf{F} 5$ and $\mathbf{F} 7$, and $D\left(s_{p_{i}}, s_{p_{j}}\right)$ is the spatial distance of the strokes, related to F1.

Variation cost. During painting, the variation from one stroke to another is affected by a changing ink or brush type, i.e., by wetness, thickness, and shape (R4-R6). We evaluate $c_{v a r}\left(s_{p_{i}}, s_{p_{j}}\right)$ according to the shape(F2-F4), and the wetness and thickness (F5) of the two brushes:

$c_{v a r}\left(s_{p_{i}}, s_{p_{j}}\right)=\frac{\left|\operatorname{sgn}(i-j)+\operatorname{sgn}\left(\sum\left(\vec{s}_{\text {var }}\left(p_{i}\right)-\vec{s}_{\text {var }}\left(p_{j}\right)\right)\right)\right|}{2} \cdot \sum\left(\vec{s}_{\text {var }}\left(p_{i}\right)-\vec{s}_{\text {var }}\left(p_{j}\right)\right)$,

where $\vec{s}_{v a r}\left(p_{i}\right)=\left(A\left(s_{p_{i}}\right), L\left(s_{p_{i}}\right), S\left(s_{p_{i}}\right), W\left(s_{p_{i}}\right), T\left(s_{p_{i}}\right)\right)$ is a vector composed of the stroke features F2-F4 and F6. This condition guarantees a cost of zero when the drawing order of the two strokes is consistent with the principles R4-R6.

As the rules of wetness, thickness and shape are only effective within strokes of the same semantics, the priority of variation cost needs to be obviously lower than consistency cost. Thus, we set $\omega=0.7$ in Equation (2) by default, with the weight of variation at only 0.3 .

Regulation term. We propose $d\left(p, p^{*}\right)$ as a regularization term [40] to preserve the stage-level stroke orders which are acquired from the topological sorting. The Spearman's rank correlation coefficient (Spearman's $\rho$ ) is adopted to measure the similarity between two stroke orders. Supposing we have $N$ strokes; the $i_{t h}$ stroke's rank in $p$ is $p_{i}$, and the regularization term is computed from following:

$$
d\left(p, p^{*}\right)=\rho=1-\frac{6 \sum d_{i}^{2}}{N\left(N^{2}-1\right)},
$$

where $d_{i}=p_{i}-p_{i}^{*}$, is the difference between ranks.

Constraints. As an important effect for coupling strokes, guideline $\mathbf{R} 7$ implies that strokes representing a round object must be added together. To guarantee that the optimal order agrees with R7, we traverse the stroke set to find any couple of strokes representing a round object. In our experiments we consider two strokes $s_{i}$ and $s_{j}$ to represent such a round object if $D\left(s_{i}, s_{j}\right)$ is less than $10 \%$ of the image width and $\frac{D^{\prime}\left(s_{i}, s_{j}\right)}{D\left(s_{i}, s_{j}\right)}<1.2$. The permutation should guarantee that such a couple of strokes is always added together. This information is formulated as a hard constraint within the global minimization problem.

\subsection{Optimization of stroke ordering}

In [15], stroke order optimization is solved by finding the Hamiltonian path in a directed graph and using the branchand-bound approach for pruning. However, our problem 
is more complex than the problem in [15] because of the regularization term and the encoding of the consistency cost and variation cost in our energy function. Consequently, the optimization method in [15] is unsuitable for our task.

To obtain the optimal order efficiently and accurately, we adopt NES [5], a family of black-box optimization methods, to search for a solution.

Encode R7. The energy function in Section 5.3, including the given hard constraints, is not appropriate to solve by NES. Inspired by the work of Liu et al. [41], who add labeling information (hard constraints) to an unsupervised learning algorithm and arrive at a semi-supervised nonnegative matrix factorization, we transform the problem of stroke sorting to order a set of stroke groups. Two strokes representing one round object are put into one group; otherwise, one stroke is put into in one group. We apply NES to order the stroke groups. The feature of the stroke group is the average of the members in one stroke group. The order of the strokes within one group is simply determined by referring to $\sum \vec{s}_{\text {var }}$ in Section 5.3. Thus, we can obtain an order of all strokes $(p)$ from the permutation of the corresponding stroke pairs.

Encoding space. In applying NES, the permutation problem has to be encoded into a real-valued space because finding a parameterized search distribution that represents the structure of the solutions is difficult in a permutation space. Ceberio et al. [42] discuss two types of encoding modes for encoding permutation problems into a real-valued space. Inspired by modes based on random keys, we encode the permutation of $M$ stroke groups in the following way. Each stroke group is assigned a from some real domain (an integer number in the range of $[1, M]$ ). The value $\in \mathbb{N}^{M * 1}$ for all stroke groups is also obtained. Subsequently, the groups are sorted according to the value to obtain the resulting permutation. For each stroke, the assigned value should obey a normal distribution.

A multi-normal case of exponential natural evolution strategies (xNES) is now adopted to find the optimized solution of Equation (2). The pseudocode for applying xNES to solve our problem is shown in Algorithm 1 where $\mathbb{I}=\operatorname{diag}(1, \ldots, 1) \in \mathbb{R}^{M * M}$ and $f(p)$ is the goal of optimization. The xNES is updated by making use of Adaptation Sampling (AS) in [5].

Initial solution and parameter setting. The $x N E S$ requires two inputs to describe the initial solution: $\mu_{\text {init }}$ represents the initial mean vector of the multinomial distribution and covariance factor $A$ depicts the covariance information. Although the influence of initialization does not last long (i.e., even a bad initial solution can yield a reasonable result after numerous iterations), we find that using the result of the permutation of the stroke groups, which is transformed from topological ordering as $\mu_{\text {init }}$, leads to a rapid convergence of the iteration process. The computation time for optimization is related to the number of the stroke groups and ranges from a few seconds (14 strokes) to less than four minutes. The default parameters are set in Table 1.

\subsection{Dynamically drawing strokes}

Stroke direction. Determining the direction of an individual stroke is equivalent to judging which of the two outer skeletal
Algorithm 1 Exponential Natural Evolution Strategies(xNES)

Require: fitness function $f(p)=c(p)+d\left(p, p^{*}\right)$, initial solution $\mu_{\text {init }}$, covariance factor $A$

1: initial $\sigma \leftarrow \sqrt{|\operatorname{det}(A)|}, \mathcal{B} \leftarrow A / \sigma$

2: repeat

for $k=1 . . \lambda$ do

draw sample value ${ }^{k} \sim \mathcal{N}(0, \mathbb{I})$

value $^{k} \leftarrow \mu+\sigma \mathcal{B}^{T}$ value $e^{k}$

sort value $e^{k}$ and get the permutation $p^{k}$

evaluate the fitness $f\left(p^{k}\right)$

end for

sort $\left\{\left(\right.\right.$ value $\left.\left.e^{k}, p^{k}\right)\right\}$ with respect to $f\left(p^{k}\right)$

Compute gradients $\nabla_{\delta} J, \nabla_{\sigma} J, \nabla_{\mathcal{B}} J$

Update parameters $\mu, \sigma, \mathcal{B}$ :

$\mu \leftarrow \mu+\eta_{\mu} \cdot \sigma \mathcal{B} \cdot \nabla_{\delta} J$

$\sigma \leftarrow \sigma \cdot \exp \left(\eta_{\sigma} / 2 \cdot \nabla_{\sigma} J\right)$

$\mathcal{B} \leftarrow \mathcal{B} \cdot \exp \left(\eta_{\mathcal{B}} / 2 \cdot \nabla_{\mathcal{B}} J\right)$

5: until stopping criterion is met

TABLE 1

Default parameters for XNES in Algorithm 1

\begin{tabular}{ll}
\hline Parameter & Default Value \\
\hline$\lambda$ & $2 *(4+[3+\log (M)])$ \\
$A$ & $\mathbb{I}$ \\
$\eta_{\mu}$ & 1 \\
$\eta_{\sigma}$ & $\frac{9+3 \log (M)}{5 M \sqrt{M}}$ \\
$\eta_{\mathcal{B}}$ & $\frac{3+3 \log (M)}{5 \sqrt{M}}$ \\
\hline
\end{tabular}

points is the starting point. In accordance with the guidelines in Section 4.3 (D1, and D2), the skeletal point preferred as the first point is measured by the average ink wetness and thickness of pixels nearby.

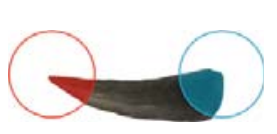

(a) Stroke direction

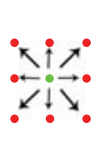

(b) Connected domains
Fig. 9. Stroke direction and connected domains.

Animation. The last step is to dynamically display the stroke sequence given the permutation of a set of strokes and the direction of each stroke. Inspired by the flood filling algorithm, we take the start point of an individual stroke as the initial seed point. The process of reproducing the stroke is treated as obtaining new seeds from the footprints of the stroke. For thin strokes (scale of less than 30 before being normalized), the eight-connected domain (see Fig. 9(b) left) is used to probe the new seed. The twelve-connected domain (see Fig. 9(b) right) is used for the remaining strokes. The display speed can be controlled by adjusting the refresh frequency. When drawing the stroke with a certain refresh frequency, the speed is attenuated with the increment of the stroke width. In our system, we use an ellipse-shaped footprint model to improve the visual effect. By finding the minimum bounding ellipse of the new footprints during each refresh, we yield the results that are similar to those of previous methods (see Fig. 10). 


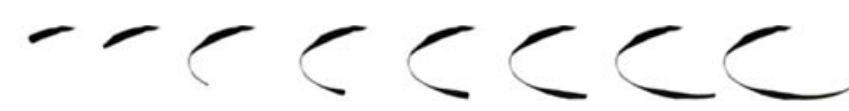

Fig. 10. Dynamically drawing a stroke.

\section{Results and Discussion}

We implement our system on a PC with $3.4 \mathrm{GHz}$ Intel Core i7 and 16 GB DDR3 memory. After all the strokes are obtained, the calculation time of stroke ordering ranges from 10 seconds to 4 minutes, depending on the number of strokes in a painting.

\subsection{Evaluation}

Dataset. Traditional Chinese brush painting involves basic imageries that are widely used to introduce painting skills. Our painting image set (see Fig. 11 top), which contains 19 input ink-wash paintings, overlays the following imageries: landscape painting (Example 7), bird-and-flower painting (Examples 2, 4, 5, 11, 13 and 15), insect-and-fish painting(Examples 3 and 17), and some other imageries that usually appear in the tutorial of Chinese brush paintings. Some parts of the paintings were drawn by artists we invited, and the rest are the masterpieces of the masters such as Qi Baishi (http://www.chinaonlinemuseum.com/ painting-qi-baishi.php).

Experimental settings. For each painting, we invited six artists with more than seven years of painting experience to manually sort strokes A1-A6 though an online game (see supplemental materials).

To evaluate the soundness and effectiveness of the proposed guidelines, we apply a quantitative evaluation by comparing our results with three "weak orders" and the order from the artists. Both the multi-stage structure and guidelines are encoded into the energy equation as consistency cost, variation cost, and regulation term. We generate three weak orders for each painting by ignoring one of the three parts in Equations (2), (3), and (6): W1 by removing the consistency cost from the energy function, W2 by removing the variation cost, and $\mathbf{W} 3$ by removing the regulation term.

We likewise conduct a user study to evaluate the perceived quality of our solutions compared with alternative strategies. We choose three baseline methods for comparison: the topological ordering Topo (Section 5.3), the order submitted to Fu et al.'s method [15] in which the branch-andbound $\mathbf{B} \& \mathbf{B}$ algorithm serves as the optimizer, and thickestink-first ordering TFO which is used in Yang et al. [18]. To use the mechanism by Fu et al. [15], who built a two-level representation of a painting and optimize the order in each level, we first split all strokes into two sets: zero-in-degree strokes in the Hasse diagram are considered in the primary level, whereas the others are considered in the detail level. Then, we apply branch-and-bound search to each level to find an optimized order of Equation (2). Fig. 12 shows the reconstruction results of paintings 1, 4, 9, and 18 generated by different methods. For consistency, the scheme of stroke direction determination shown in Section 5.5 is used for all methods when generating animation.

Experiment I. We evaluate the degree of consistency between our results and the results of artists relative to that of W1-W3 and the artist'. The rank correlation coefficient (see Equation 6) is used to measure the consistency of two ordered lists. As shown in Fig. 13, for each pair under comparsion, we calculate Spearman's $\rho$ to measure the similarity between two orders for each painting and show the average correlation coefficients. Intuitively, the Spearman correlation between two subjects(methods) will be high when observations have a similar rank and low when observations have a dissimilar rank. The results in Fig. 13 indicate the following:

- Although all artists are skilled in ink-wash painting, their results are not absolutely consistent (none of the average correlation coefficients equals 1 ). Nevertheless, they are all significantly correlated. Such observation confirms the main motivation throughout our work: although the drawing process is individual and involves personal preferences, artists generally follow certain principles because of some conventions. This condition guarantees that an order created by a computer is sensible and usable in teaching Chinese brush paintings.

- The average correlation coefficients between our results and those of other artists are significantly correlated, and the coefficients show that our algorithm can generate reasonable solutions that are consistent with those for well-trained painters.

- The average correlation coefficients between the three week orders and the artists are not significantly correlated. This lack of correlation means that the results generated without a multi-stage structure or consistency/variation cost are not consistent with those of other artists. Such observation implies that the multi-stage structure and guidelines are necessary for solving this problem.

The quantitative evaluation and statistics above suggest can be made that the proposed approach can generate reasonable results that are consistent with those of artists.

Experiment II. We evaluate the relative effectiveness of our algorithm compared with three baseline methods: Topo, B\&B, and TFO. For each stimulus, we first ask a participant to watch two anonymous animations, one is generated by our method and the other is randomly picked from the results of the three contrast methods. We then ask the participant to choose the result that is more reasonable. Ten examples are randomly picked from the image set for each participant. A total of 63 participants (34 males, 29 females, age range of 2045) from different backgrounds, age groups and backgrounds attended the comparison of 10 pairs of animations. Most of the participants had been well trained in Chinese painting, and some of them are reported to be artists. Each example is evaluated by more than 30 participants on average, and our algorithm is compared with each local method by more than 200 times. Table 2 shows the statistics. Each row shows the competition between our method and the one indicated on that row. The mean of the comparisons shows the average times our method is compared with the other methods on each image. The mean of wins indicates the number of times (or percentage) our method wins the competitions for each image. From the statistics, our method generally outperforms all competitors. Fig. 11(a) shows the normalized votes for the five methods. For paintings with low complexity and few 


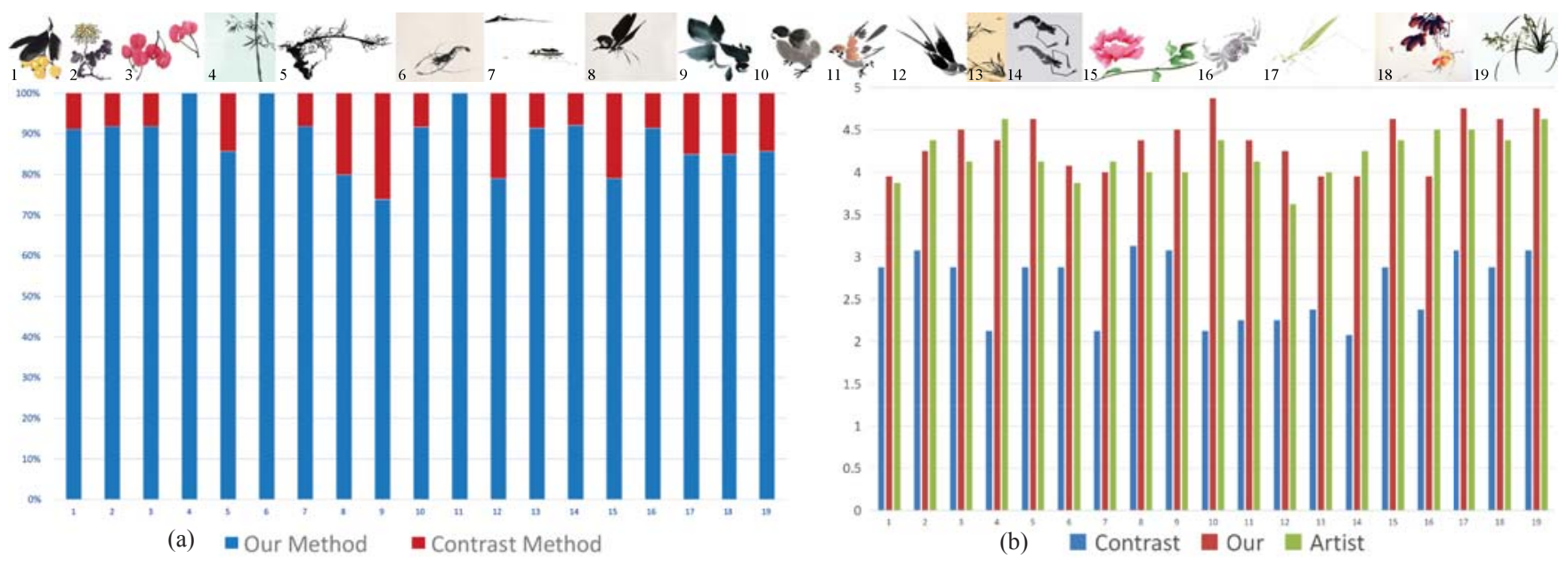

Fig. 11. Experimental results. Left: normalized votes for our method and average of the three contrast methods in II. Right: mean DA for individual orders made by the best local method, our method, and one of the artists in Experiment III.

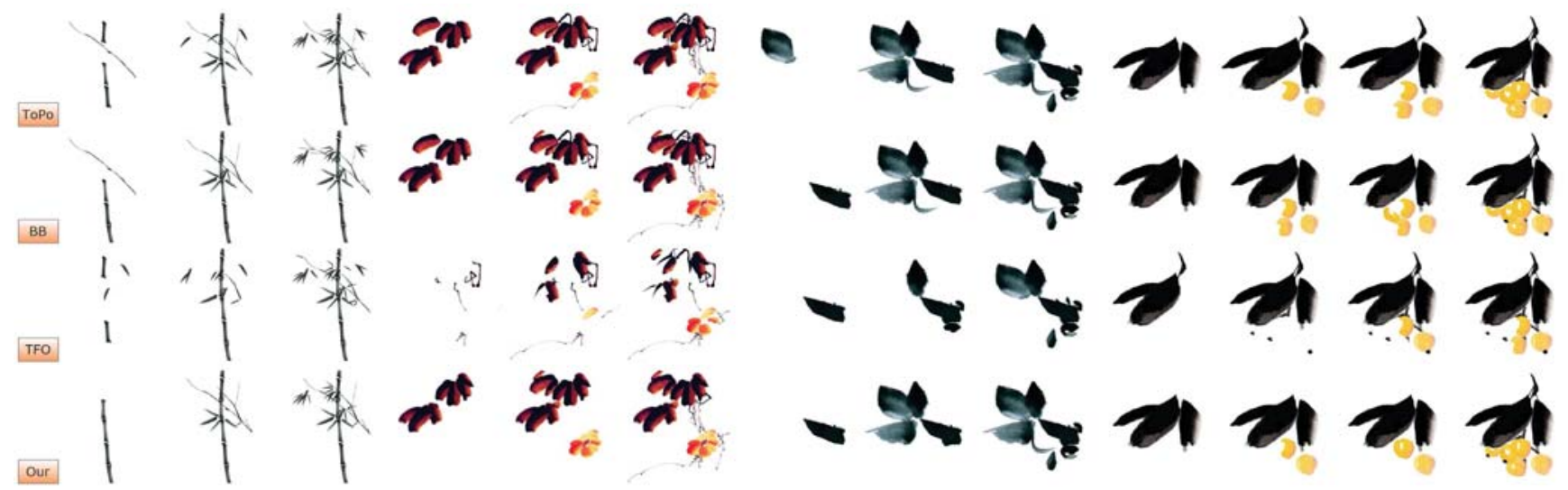

Fig. 12. Screenshots after drawing the input paintings 1,4,9, and 18 with the same number of strokes using different methods. Our results are on the lowest row.

TABLE 2

User study results II. The first column indicates the type of the local method, and the second column shows the average number of our method was compared with the other methods.

\begin{tabular}{|c|c|c|c|c|c|}
\hline Contrast & \multirow{2}{*}{$\begin{array}{c}\text { Mean of } \\
\text { Methods }\end{array}$} & Mean (\%)of & \multirow{2}{*}{ Std.dev } & \multicolumn{2}{|c|}{ 95\% confidence interval } \\
\cline { 5 - 6 } & Comparisons & wins & & Lower Bound & Upper Bound \\
\hline ToPo & 10.95 & $9.10(84 \%)$ & 0.84 & 8.68 & 9.53 \\
\hline BB & 11.05 & $8.94(80 \%)$ & 0.92 & 8.49 & 9.41 \\
\hline TFO & 11.16 & $10.10(90 \%)$ & 0.46 & 9.87 & 10.34 \\
\hline
\end{tabular}

strokes (Example 9), the contrast methods also perform well. The reason is that for those images, the differences among the orders computed by different methods are not overly significant.

Experiment III. In this experiment we evaluate our method among other subjects to determine whether the results retrieved by our method could be used in a tutorial on Chinese brush painting. For each painting, we randomly pick one result from the artists' as reference and choose the best performing local method as a contrasting method. The participants were requested to rate the degree of accuracy of an order on a discrete scale from 1 to 5 , where 1 means completely wrong and 5 means completely right. Fig. 11(b) plots the mean and the corresponding standard deviation for individual orders, based on the basis of the feedback from 40 participants with ages between 30 and 44 years(all with artistic training) for each example. First, we test the interrater reliability to verify the objectivity of the collected scores. Kendall's tau $(\tau)$ coefficient is usually used to measure the reliability coefficient when more than two raters are involved. The results of $\tau=0.681$ and sig. $=5.44 e^{-14}$ confirm that the subjects have a general consensus with regard to the rating of the degree of accuracy. Then, we perform a one-sample $t$-test (test value $=4$ ) on the scores of our method to verify the accuracy of our results. The results show that no significant difference ( $p$-values $>0.05$ ) between the mean value of the scores for our results and the value of 4 , which in the range of scores means "approximately correct". A paired $t$-test on the scores between our method and those by other artists shows no significant difference ( $p$-values $>0.05)$ between the two sets of results for most of the models. The results proved that our method has the ability to find a reasonable solution. As expected, a paired $t$-test between our method and the best local method shows significant difference between the two 


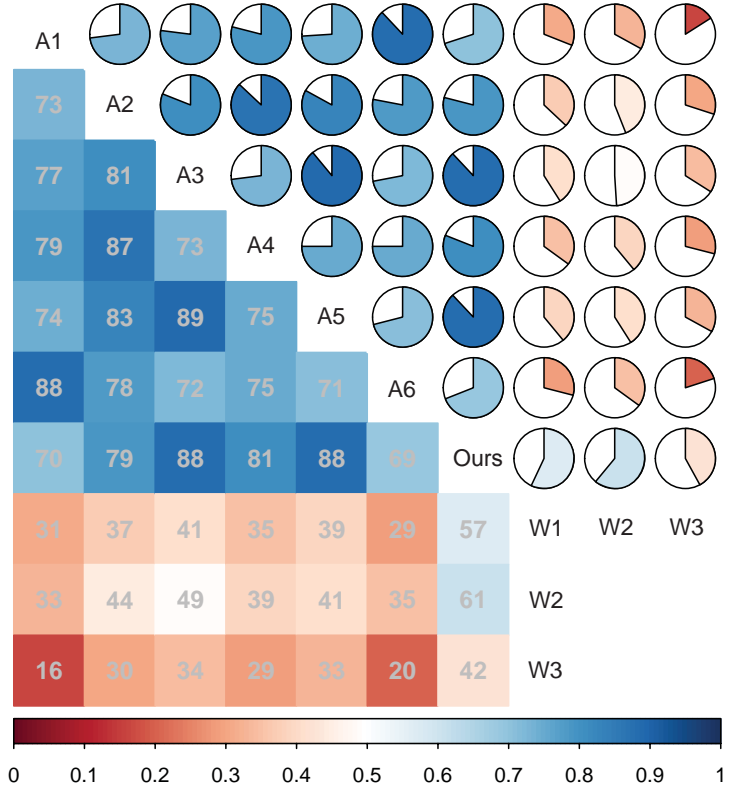

Fig. 13. Correlation among different rankings. For clarity, the results are shown in two visual ways. The lower left part of the figure shows the average values in percentage and the upper right part shows the values in a fan chart.

methods for most of the models.

\subsection{Applications}

Education. In learning Chinese brush painting, one of the most important parts is to understand the composition process of a painting. Therefore, in a painting class students often practice by following the teacher in drawing a painting stroke by stroke. Our system boosts the possibility of creating a large number of stroke sequences from various paintings that might help to teach good ordering of strokes. We also develop an interactive mobile application (APP.) to help people understand the creation process of brush paintings (see Fig. 14). The main interface of our APP. displays a painting with all strokes initially set to gray. Then, the system highlights the boundaries of the strokes one by one. The user needs to slide across the current highlighted stroke to unlock the next one. This APP. are useful in teaching kids some basic knowledge of Chinese brush paintings in early education before they can hold a brush for a long time to draw (which is usually at an age of 7 to 8 years). The APP. is also interesting for adults if they want to learn painting because the painting process is always the most important part for understanding such paintings.

Animation. Animation generally contains more information than a static painting. By adding strokes one by one in the determined order of our system, we can easily produce animations that simulate the process of creation. Vivid drawing animations can be produced by augmenting animations with appropriate background music. Since drawing speed attenuates with increasing stroke width [23], we slow down the drawing speed slightly for such strokes (see supplementary video).

We show the utility of our synthesized painting animations in two applications. Aside from using them for tutorial videos for teaching painting skills, our animations can also show multiple strokes at a time to generate different visual effects in commercials. Please see the supplementary video for examples of dynamic paintings. Another application is for story telling (or video scribing) via painting animation with additional narration. Previous video scribing works were created using pen-and-ink style. With our technology, this multimedia form can now be created in Chinese brush painting style (see Fig. 15). Note that for a static scene, we manually partition the individual components and assign the component order with respect to the narration.

Painting from nature. To create their paintings, artists often go to the wild to draw natural objects or scenes. Utilizing an image stylization technology such as presented by [27], we can convert real images into brush paintings (see Fig. 16). We then use our system to produce an animation to simulate the painting process from nature (see supplementary video).

\subsection{Limitations}

Developing a general method to reconstruct the drawing process of arbitrary Chinese brush painting is actually difficult because of the many unconventional cases produced by some special and complicated techniques. In some cases, semantic information becomes crucial for getting a good stroke order. For example, in flower paintings, artists often like to draw petals first, and then leaves and other elements. However, the lack of high-level information in the guidelines may cause an unnatural drawing order in our results. The main reason is that a global optimization strategy is used to order the strokes, and this strategy only formulates lowlevel, feature-based guidelines for the objective function. The lack of integration of semantic information during stroke ordering may separate some strokes that should be drawn together. Although our framework works well for paintings of small freehand style, it is still difficult to use to construct the drawing process of large impressionistic ink landscapes and other meticulous painting styles due to the difficulties in stroke extraction. And the rules are not totally suitable for handling the outline strokes.

In this work, we mainly focus on the ordering of strokes during the whole process of drawing. This focus is valid for computer-learning digital art analysis and for painting education, artwork research, and development of new art forms with computer. For the rest of the parts of the drawing process, we adopt some efficient and straightforward solutions. We build an ellipse-shaped footprint model for animation rendering. Although this method can generate compelling results, it does not take the stroking force and drawing velocity into consideration. During animation, the stroke directions are modeled roughly by the two painting preferences. However, directions may be distinctively different for different objects and may be influenced by the convenience of the mechanical movement toward the subjects wrists and fingers, as well as the visibility of existing strokes. Hence, no universal principle exists for the direction of strokes in Chinese brush paintings. No complicated physical brushes and movement models are built because they are beyond the main scope of this work. 

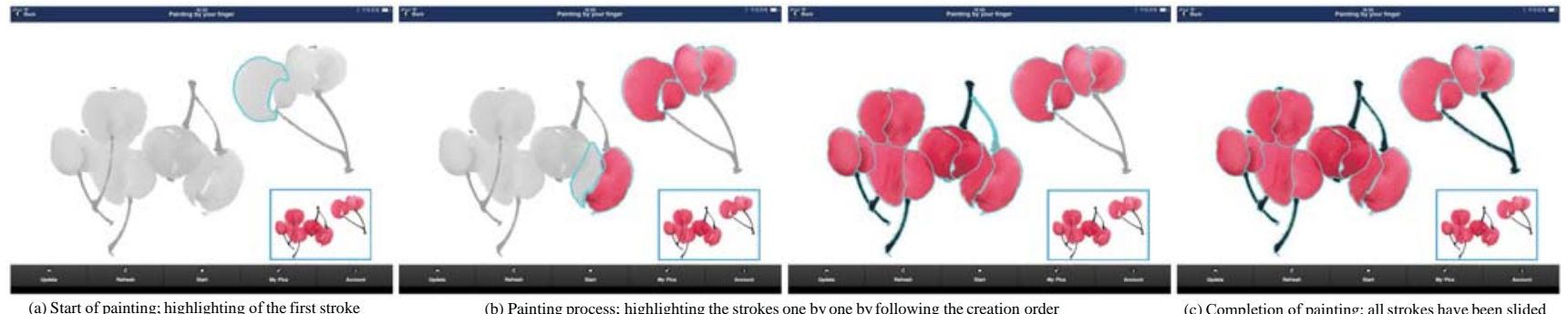

Fig. 14. Our mobile APP intends to help people understand and learn the creation of Chinese brush paintings.
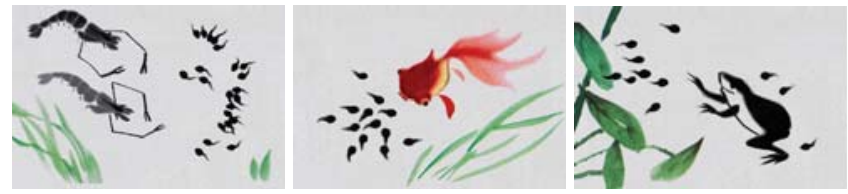

Fig. 15. Animation for vivid storytelling. See the accompanying video for the narrated animation.

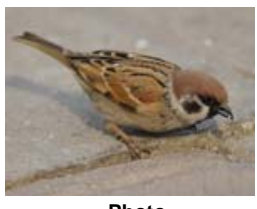

Photo

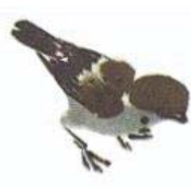

Painting

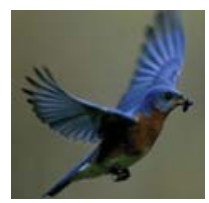

Photo

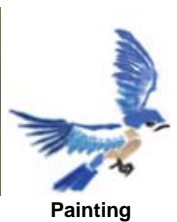

Painting
Fig. 16. Image stylization for painting process reconstruction.

\section{Conclusion AND Future Work}

We introduced the problem of the animated construction of Chinese brush paintings, and summarized the key principles of drawing order from art canons. We first define a partially ordered relation over the set of painting strokes and then construct a diagram structure to serve as a multi-stage drawing order. After encoding corresponding ordering guidelines into an energy function, we obtain an optimal ordering using a natural evaluation strategy from a topological sorting result of the Hasse diagram. We tested our framework on a range of input paintings and evaluated the results via some experiments. Lastly, we demonstrated some potential applications, especially for making tutorial videos.

In the future we will explore data-driven approaches for learning specific drawing styles of artists, which can be integrated as an additional attributes into our process to construct personalized drawing sequences. Furthermore, there are specific drawing principles for specific categories of Chinese brush paintings (insects, flowers, birds, etc.), so we will try to create more detailed guidelines to customize the construction of a drawing process according to the content of an input painting. Lastly, we want to develop a more advanced stroke rendering method for dynamically showing the rhythm of drawing.

\section{ACKNOWLEDGMENTS}

This work was supported by National Natural Science Foundation of China under nos. 61672520, 61571046, 61501464, 61372184, and by Beijing Natural Science Foundation under No. 4162056, and by CASIA-Tencent Youtu joint research project. The Titan $X$ used for this research was donated by the NVIDIA Corporation. Weiming Dong is the corresponding author.

\section{REFERENCES}

[1] N. Yeh, Chinese Brush Painting: an Instructional Guide. Ning \& Ling Chi Yeh, 1988

[2] J. Dwight, The Chinese Brush Painting Bible: Over 200 Motifs with Step by Step Illustrated Instructions. Chartwell Books, 2011.

[3] Wikipedia, "Six principles of chinese painting," http://en. wikipedia.org/wiki/Six_principles_of_Chinese_painting, 2014, online; accessed 22-October-2014.

[4] M.-M. Sze, The Tao of Painting: A Study of the Ritual Disposition of Chinese Painting. Bollingen Foundation, Pantheon Books, 1963.

[5] D. Wierstra, T. Schaul, T. Glasmachers, Y. Sun, J. Peters, and J. Schmidhuber, “Natural evolution strategies," J. Mach. Learn. Res., vol. 15, no. 1, pp. 949-980, Jan 2014.

[6] M.-M. Sze, The Mustard Seed Garden Manual of Painting. Princeton University Press, 1978.

[7] N. Yeh, 108 Flowers. Ning Yeh and Lingchi Yeh, 2013.

[8] N. Rae, The Ch'i of the Brush: Capturing the Spirit of Nature with Chinese Brush Painting Techniques. Watson-Guptill, 2003.

[9] L. Novick and B. Tversky, "Cognitive constraints on ordering operations: the case of geometric analogies," Journal of Experimental Psychology: General, vol. 116, no. 1, pp. 50-67, Mar. 1987.

[10] N. S.-H. Chu and C.-L. Tai, "Moxi: Real-time ink dispersion in absorbent paper," ACM Trans. Graph., vol. 24, no. 3, pp. 504-511, Jul. 2005.

[11] M. K. Babcock and J. J. Freyd, "Perception of dynamic information in static handwritten forms," The American Journal of Psychology, vol. 101, no. 1, pp. 111-130, 1988.

[12] J. Hays and I. Essa, "Image and video based painterly animation," in Proceedings of the 3rd International Symposium on Non-photorealistic Animation and Rendering (NPAR). New York, NY, USA: ACM, 2004, pp. $113-120$.

[13] M. Flagg and J. M. Rehg "Projector-guided painting," in Proceedings of the 19th Annual ACM Symposium on User Interface Software and Technology (UIST). New York, NY, USA: ACM, 2006, pp. 235-244.

[14] A. Monroy, B. Carque, and B. Ommer, "Reconstructing the drawing process of reproductions from medieval images," in 18th IEEE International Conference on Image Processing (ICIP), Sept 2011, pp. $2917-2920$.

[15] H. Fu, S. Zhou, L. Liu, and N. J. Mitra, "Animated construction of line drawings," ACM Transactions on Graphics, vol. 30, no. 6, pp. 133:1-133:10, Dec. 2011.

[16] J. Liu, H. Fu, and C.-L. Tai, "Dynamic sketching: Simulating the process of observational drawing," in Proceedings of the Workshop on Computational Aesthetics, ser. CAe '14. New York, NY, USA: ACM, 2014, pp. 15-22.

[17] X. Mao, X. Liu, T.-T. Wong, and X. Xu, "Region-based structure line detection for cartoons," Computational Visual Media, vol. 1, no. 1, pp. 69-78, 2015.

[18] L. Yang, T. Xu, and X. Li, "An image-based approach in animating painting procedure of chinese ink painting," in Proceedings of Computer Graphics International (CGI). Computer Graphics Society, 2011, pp. S26:1-S26:4.

[19] J. Tan, M. Dvorožňák, D. Sýkora, and Y. Gingold, “Decomposing time-lapse paintings into layers," ACM Transactions on Graphics, vol. 34, no. 4, pp. 61:1-61:10, Jul. 2015. 
[20] S. Xu, Y. Xu, S. B. Kang, D. H. Salesin, Y. Pan, and H.-Y. Shum, "Animating chinese paintings through stroke-based decomposition," ACM Trans. Graph., vol. 25, no. 2, pp. 239-267, Apr. 2006.

[21] S. Zhang, T. Chen, Y. Zhang, S. Hu, and R. Martin, "Video-based running water animation in chinese painting style," Science in China Series F: Information Sciences, vol. 52, no. 2, pp. 162-171, 2009.

[22] L. Yang and T. Xu, "Animating chinese ink painting through generating reproducible brush strokes," Science China Information Sciences, vol. 56, no. 1, pp. 1-13, 2013.

[23] L.-J. Yang, T.-C. Xu, X.-S. Li, and E.-H. Wu, "Feature-oriented writing process reproduction of chinese calligraphic artwork," in SIGGRAPH Asia 2014 Technical Briefs, ser. SA '14. New York, NY, USA: ACM, 2014, pp. 5:1-5:4.

[24] J. Lee, "Simulating oriental black-ink painting," IEEE Computer Graphics and Applications, vol. 19, no. 3, pp. 74-81, May 1999.

[25] C. Amati and G. J. Brostow, "Modeling 2.5d plants from ink paintings," in Proceedings of the Seventh Sketch-Based Interfaces and Modeling Symposium, ser. SBIM '10. Aire-la-Ville, Switzerland, Switzerland: Eurographics Association, 2010, pp. 41-48.

[26] C.-M. Wang and R.-J. Wang, "Image-based color ink diffusion rendering," IEEE Transactions on Visualization and Computer Graphics, vol. 13, no. 2, pp. 235-246, March 2007.

[27] N. Xie, H. Hachiya, and M. Sugiyama, "Artist agent: A reinforcement learning approach to automatic stroke generation in oriental ink painting," in Proceedings of the International Conference on Machine Learning (ICML), 2012, pp. 153-160.

[28] T.-C. Xu, L.-J. Yang, and E.-H. Wu, "Stroke-based real-time ink wash painting style rendering for geometric models," in SIGGRAPH Asia 2012 Technical Briefs, ser. SA '12. New York, NY, USA: ACM, 2012, pp. 19:1-19:4.

[29] L. Dong, S. Lu, and X. Jin, "Real-time image-based chinese ink painting rendering," Multimedia Tools and Applications, vol. 69, no. 3, pp. 605-620, 2014

[30] F. Yao and G. Shao, "Painting brush control techniques in chinese painting robot," in IEEE International Workshop on Robot and Human Interactive Communication, Aug 2005, pp. 462-467.

[31] T. Lindemeier, S. Pirk, and O. Deussen, "Image stylization with a painting machine using semantic hints," Computers $\mathcal{E}$ Graphics, vol. 37, no. 5, pp. 293-301, Aug. 2013.

[32] O. Deussen, "e-David painting robot," http://www.informatik. uni-konstanz.de/en/edavid/, 2012.

[33] Y. Sun and Y. Xu, "A calligraphy robot - Callibot: Design, analysis and applications," in IEEE International Conference on Robotics and Biomimetics (ROBIO), Dec 2013, pp. 185-190.

[34] F. Tang, Y. Meng, W. Dong, F. Huang, F. Huang, and X. Zhang, "Animated construction of ink-wash paintings," in SIGGRAPH Asia Posters, 2014, pp. 23:1-23:1.

[35] J. B. Tenenbaum, V. Silva, and J. C. Langford, "A Global Geometric Framework for Nonlinear Dimensionality Reduction," Science, vol. 290, no. 5500, pp. 2319-2323, 2000.

[36] Q. Yan, L. Xu, J. Shi, and J. Jia, "Hierarchical saliency detection," in Proceedings of the IEEE Conference on Computer Vision and Pattern Recognition (CVPR). Washington, DC, USA: IEEE Computer Society, 2013, pp. 1155-1162.

[37] S. S. Khan and D. Vogel, "Evaluating visual aesthetics in photographic portraiture," in Proceedings of the Eighth Annual Symposium on Computational Aesthetics in Graphics, Visualization, and Imaging, ser. CAe '12. Aire-la-Ville, Switzerland, Switzerland: Eurographics Association, 2012, pp. 55-62.

[38] J. Bang-Jensen and G. Z. Gutin, Digraphs: Theory, Algorithms and Applications, 2nd ed. Springer Publishing Company, Incorporated, 2008.

[39] S. Radhakrishnan, D. Kolippakkam, and V. S. Mathura, Introduction to Algorithms (3rd ed.). Springer US, 2009.

[40] D. Wang, S. C. Hoi, P. Wu, J. Zhu, Y. He, and C. Miao, "Learning to name faces: A multimodal learning scheme for search-based face annotation," in Proceedings of the 36th International ACM SIGIR Conference on Research and Development in Information Retrieval, ser. SIGIR '13. New York, NY, USA: ACM, 2013, pp. 443-452.

[41] H. Liu, Z. Wu, X. Li, D. Cai, and T. Huang, "Constrained nonnegative matrix factorization for image representation," IEEE Transactions on Pattern Analysis and Machine Intelligence, vol. 34, no. 7, pp. 1299-1311, July 2012.

[42] J. Ceberio, A. Mendiburu, and J. A. Lozano, "A preliminary study on edas for permutation problems based on marginal-based models," in Proceedings of the 13th Annual Conference on Genetic and
Evolutionary Computation, ser. GECCO '11. New York, NY, USA: ACM, 2011, pp. 609-616.

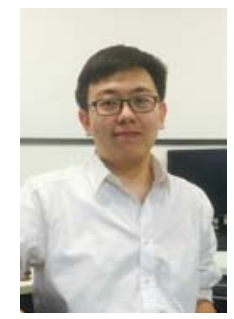

Fan Tang received the BSc degree in computer science from North China Electric Power University in 2013. He is currently working toward the PhD degree in National Laboratory of Pattern Recognition, Institute of Automation, Chinese Academy of Sciences. His research interests include computer graphics, computer vision and machine learning.

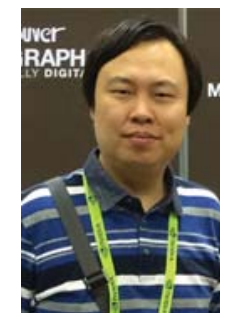

Weiming Dong is a Professor in the SinoEuropean Lab in Computer Science, Automation and Applied Mathematics (LIAMA) and National Laboratory of Pattern Recognition (NLPR) at Institute of Automation, Chinese Academy of Sciences. He received his BSc and MSc degrees in Computer Science in 2001 and 2004, both from Tsinghua University, China. He received his $\mathrm{PhD}$ in Computer Science from the University of Lorraine, France, in 2007. His research interests include image synthesis and image recognition. Weiming Dong is a member of the ACM and IEEE.

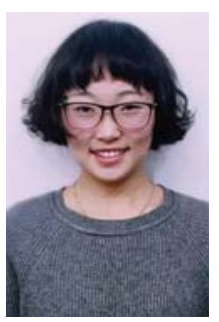

Yiping Meng received the BSc degree in Software Engineering from University of Electronic Science and Technology of China in 2013. She is currently working toward the MEng degree in National Laboratory of Pattern Recognition, Institute of Automation, Chinese of Sciences. Her research interests include computer vision, image processing, and information visualization.

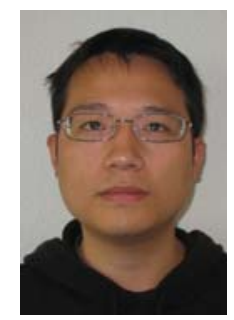

Xing Mei is an Assistant Professor in the SinoEuropean Lab in Computer Science, Automation and Applied Mathematics (LIAMA) and National Laboratory of Pattern Recognition (NLPR) at Institute of Automation, Chinese Academy of Sciences (CASIA). He received his BSc degree in Electronic Engineering in 2003 from the University of Science and Technology of China (USTC). $\mathrm{He}$ received his $\mathrm{PhD}$ degree in 2009 from CASIA. His research interests include image processing, computer vision and computer graphics.

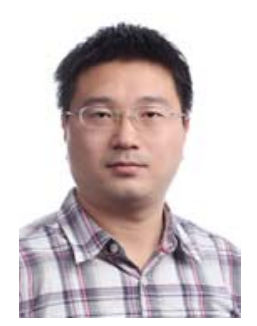

Feiyue Huang is the director of Social Network Platform Department, Tencent. He received his $\mathrm{BSc}$ and $\mathrm{PhD}$ degrees in Computer Science in 2001 and 2008, both from Tsinghua University, China. His research interests include image understanding and face recognition.

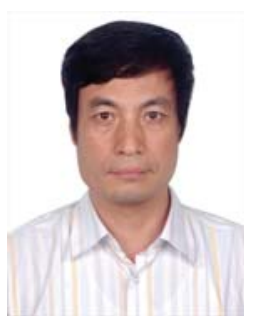

Xiaopeng Zhang received his MSc degree in Mathematics from Northwest University in 1987, and the PhD degree in Computer Science from Institute of Software, Chinese Academy of Sciences (CAS), in 1999. He is a Professor in the Sino-European Lab in Computer Science, Automation and Applied Mathematics (LIAMA) and (National Laboratory of Pattern Recognition) at Institute of Automation, Chinese Academy of Sciences (CASIA). His main research interests are computer graphics and pattern recognition. He received the National Scientific and Technological Progress Prize (Second Class) in 2004. Xiaopeng Zhang is a member of ACM and IEEE. 


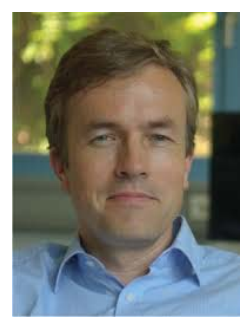

Oliver Deussen graduated at Karlsruhe Institute of Technology in 1996 and worked as a postdoctoral researcher at University of Magdeburg on Non-Photorealistic Rendering. In 2000 he was appointed as a professor for Computer Graphics and Media Design by Dresden University of Technology, since 2003 he professor for Computer Graphics and Media Informatics at University of Konstanz. He is interested in a number of areas in computer graphics and information visualization. $\mathrm{He}$ has published several books and over 100 refereed publications, he is member of ACM Siggraph, Eurographics and Gesellschaft fuer Informatik, he is associate editor of Computer Graphics Forum and Informatik Spektrum (German Journal for Computer Science). He organized several conferences, was papers co-chair of Eurovis 2004, Eurogrpahics Symposium of Rendering 2005, NPAR 2007, Computational Aesthetics 2009 and papers Co-chair of Eurographics 2011. 